\title{
Transport Equations for a Microbial Predator-Prey Community
}

\author{
E. VILLARREAL
}

Department of Physics

University of Los Andes

Bogota, Colombia

\author{
R. R. CANAle \\ Department of Civil Engineering \\ University of Michigan \\ Ann Arbor, Michigan \\ AND \\ Z. AKCASU \\ Department of Nuclear Engineering, \\ University of Michigan \\ Ann Arbor, Michigan
}

\begin{abstract}
A transport equation is used which describes the temporal behavior of interacting populations in changing environments. The formulation takes into account the internal state variables of the individuals. The general theory is applied to the transient analysis of a microbial predator-prey system using an approximate model for the specific cell growth rate and multigroup formulism to approximate the mass distribution within the population. Experimental results in a Tetrahymena pyriformis-Aerobacter aerogenes system have been used to evaluate the group parameters and test the validity of the theoretical predictions.
\end{abstract}

\section{Introduction}

There is a trend in biology toward more quantitative theories. Models are being introduced to explain the effect of variables such as temperature, $\mathrm{pH}$, and diffusion in microbial processes. These models have been partially successful in explaining experimental data but apply only to specific conditions. Models such as those used by Canale et al [1] and Curds [2] do not consider structure in the cell population. That is, small cells metabolize at the same rate as large cells. In an earlier paper [3] we introduced a multigroup theory for the population dynamics of a microbial community that takes into account the variation in the internal variables which characterize the state of an individual cell as well as the effect of the surrounding environment. In this theory the interaction between several species is expressed by a set of coupled non-linear partial differential equations. 
In the present paper this formulism is applied to a predator-prey system in which sucrose is the limiting nutrient for the growth of the bacterium Aerobacter aerogenes which, in turn, serves as the prey to the protozoan Tetrahymena pyriformis. The individual protozoan cells are characterized by their mass. The resulting equations that describe the system are solved using a multigroup approach. The individual cell mass span is divided into groups and an equations written for each group. The multigroup parameters necessary for the calculations are evaluated from experimental observations in this and in another paper [4]. The results of comparing the experimental data with the numerical calculations for two batch reactors are presented here. In one reactor the protozoan inoculum consisted of large cells, in the other it consisted of small cells.

\section{Materials and Methods}

Three experiments of the batch type were performed similar to the ones reported by Canale et al [1]. The growth medium was a solution of inorganic salts, cerophyl, and sucrose prepared in a contaminant free atmostphere. Sucrose was the limiting nutrient for the growth of the bacterium Aerobacter aerogenes which, in turn served as the prey for the protozoan Tetrahymena pyriformis. About $1500 \mathrm{~cm}^{3}$ of medium were pumped into a sterile reactor vessel, and air was bubbled through at a rate of $12 \mathrm{~cm}^{3} / \mathrm{sec}$ to assure adequate oxygen supply to the growing cells. A pH of 7.3 and a temperature of $25^{\circ} \mathrm{C}$ were maintained constant throughout the experiments. A magnetic stirrer assured a completely mixed medium. Samples were taken at intervals from one hour to several hours, the frequency varying according to the phase of growth. Once a sample was taken with a sterile syringe, an approximate protozoan mass distribution (number of ciliates in five different size groups) was obtained by using an electronic particle counter which had been calibrated using particles of known size. Centrifugation was used to separate the predator from the prey before determing the bacterial optical density at $600 \mu \mathrm{m}$. To determine the limiting nutrient concentration, samples were passed through a $0.45 \mu \mathrm{m}$ filter to remove all cells and debris, and the carbon content determined using a direct injection carbon analyzer.

Three reactors were filled and allowed to stand for several days to assure a medium free of contamination. Using a sterile syringe, a reactor (I) was inoculated with the prey bacterium. After a short lag period the prey grew exponentially and reached a peak after most of the organic carbon had been consumed. At this point the predator protozoan was inoculated into the reactor and its growth followed during the initial lag and subsequent exponential growth. During this growth period the predators consumed the prey, and after the bacterial population had been almost totally exhausted the total protozoan mass reached a maximum. The peak in the number of protozoa occurred after the peak in total mass. Therefore, after the maximum protozoan mass was reached, the decline is made at the expense of cell size during a time when protozoan number is at a maximum. Finally, the number of protozoa declined due to death of individual cells. It was 
noted that the total soluble carbon content increased steadily after the reactor had been inoculated with the predator. The largest proportion of large cells in the protozoan population occured just before the maximum in total protozoan mass in the reactor. At this time a sample was taken and inoculated into a second reactor (II) in which the bacterial population was at a maximum. This reactor had been inoculated several hours previously with bacteria, and the peak in bacterial concentration was made to coincide with the time of predator inoculation. Fig. 1 shows the behavior of the total population in this reactor and Fig. 2 through 6 show the behavior of the protozoan population in each of the five mass groups. In all three batch experiments there were large fluctuations in the initial behavior of the predators and it was also noted that just before the protozoa were inoculated into the reactors there were some counts recorded on the particle counter due to lumping of bacteria which were present at very high densities. The growth of both species in reactor II was very similar to the growth in reactor I. A third batch reactor (III) was inoculated with bacteria near the end of batch run I. At this time the protozoan population in reactor I consisted mostly of small protozoan cells. When the bacterial concentration reached a maximum in reactor III, small protozoan cells were inoculated into it. The behavior of the total populations in this reactor is shown in Fig. 7; Figs. 2 through 6 show the detailed behavior of the protozoan mass groups. As before, the overall characterisitics of the cellular behavior are not very different from reactors I and II.

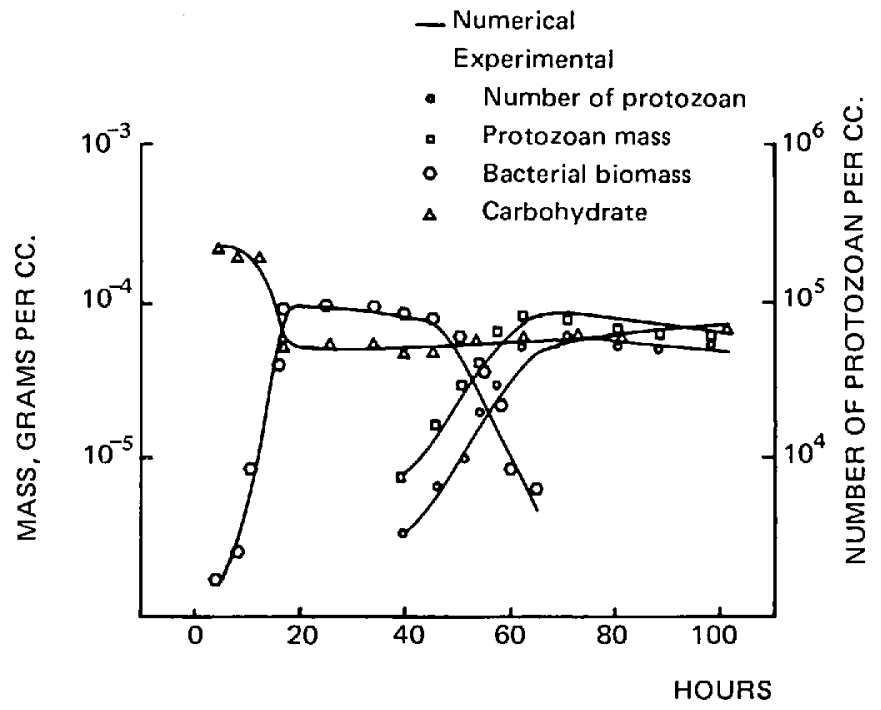

Fig. 1. Experimental and model response of batch reactor II. 


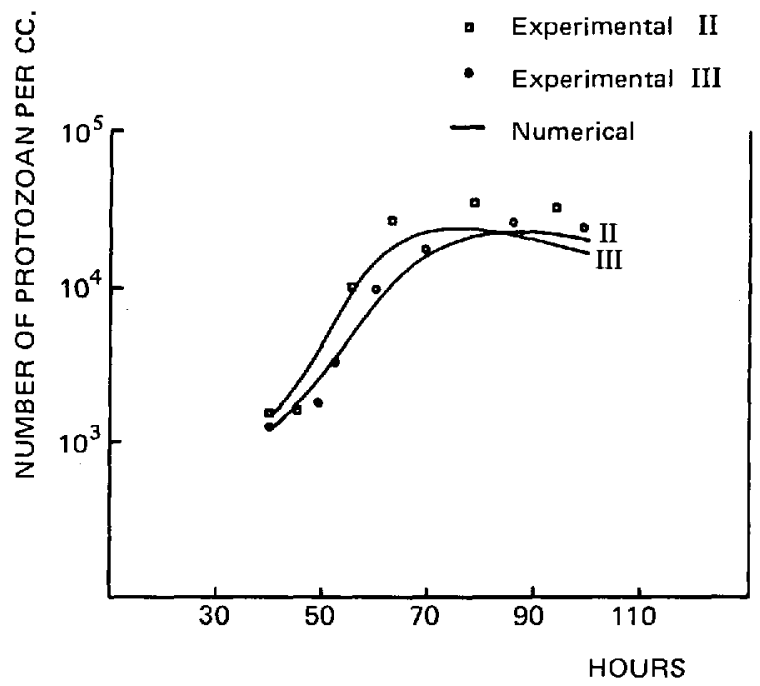

Fig. 2. Experimental and model response of protozoan in group 1 (small cells).

- Experimental II

- Experimental III

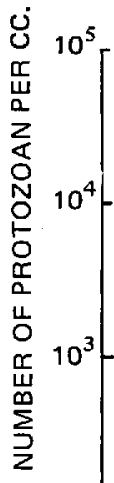

- Numerical 


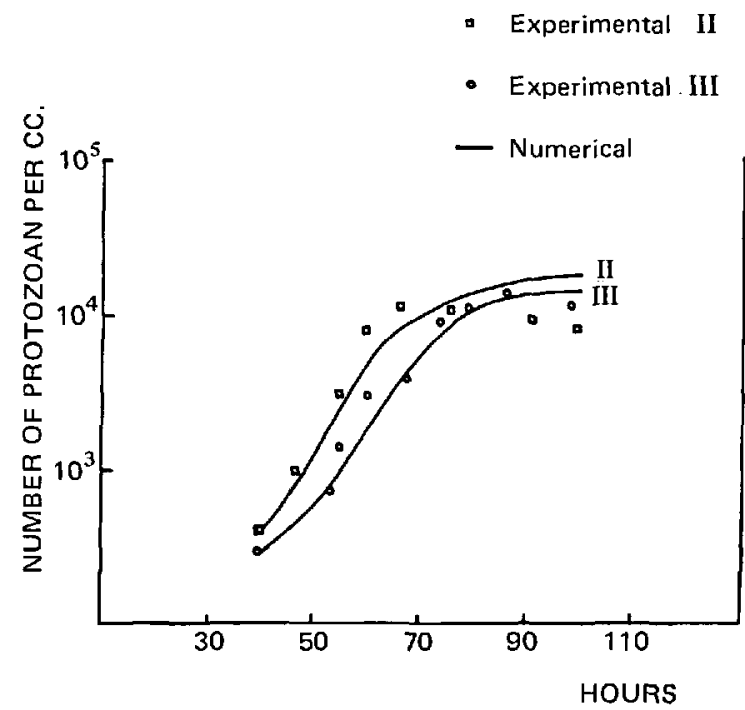

Fig. 4. Experimental and model response of protozoan in group 3.

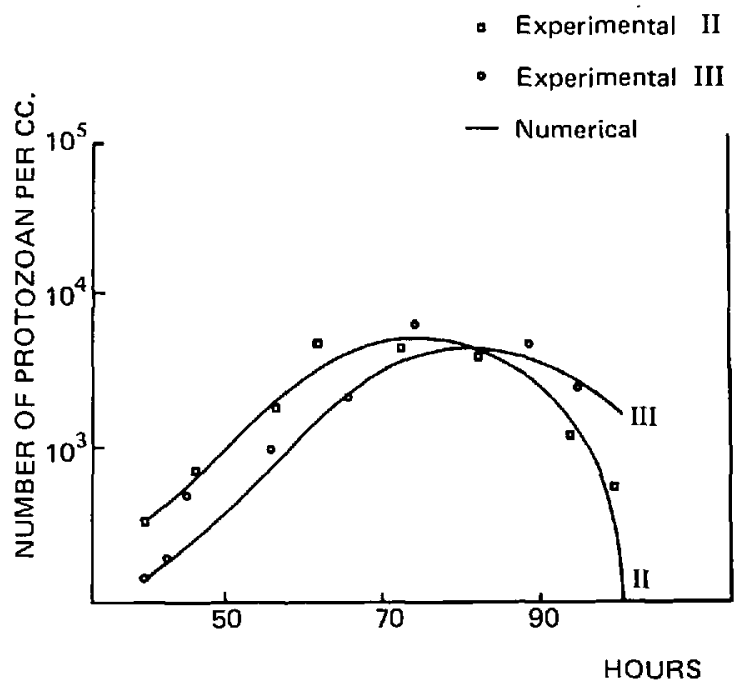

Fig. 5. Experimental and model response of protozoan in group 4. 


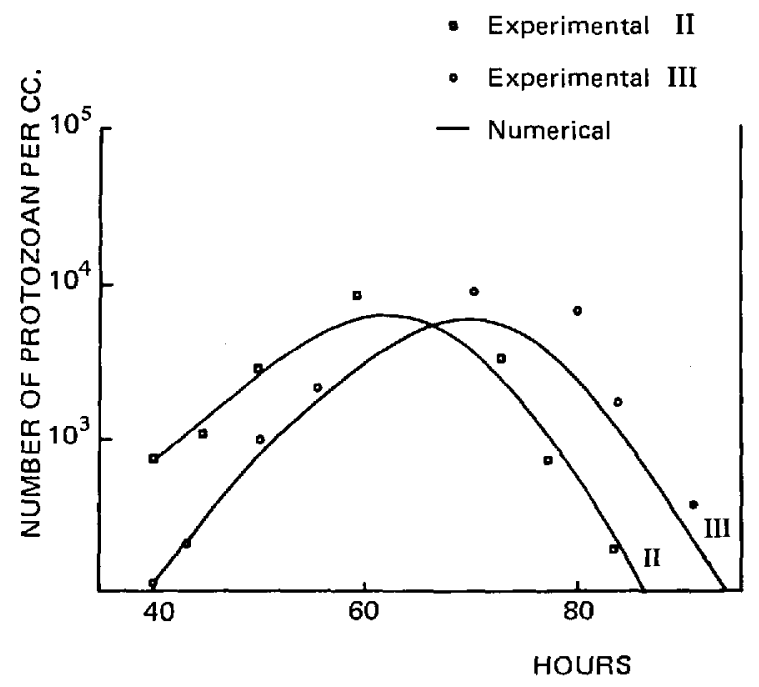

Fig. 6. Experimental and model response of protozoan in group 5 (large cells).

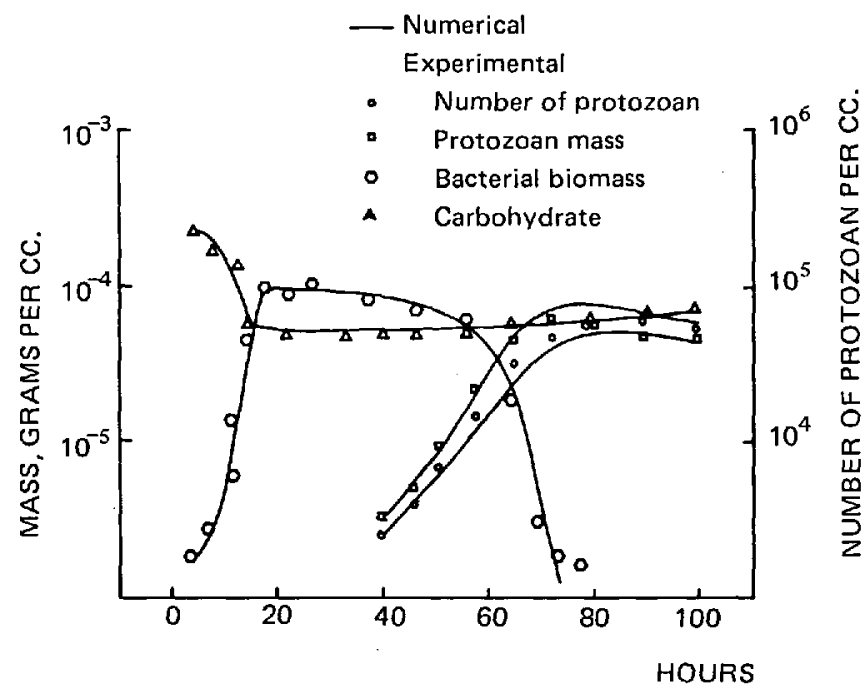

Fig. 7. Experimental and model response of batch reactor III. 


\section{Multigroup Equations}

In order to interpret the experimental results of the batch reactors quantitatively, we use the following prey-predator model which was derived in our previous papers $[3,4]$ :

$$
\begin{aligned}
\frac{d M_{p}^{g}(t)}{d t}= & {\left[m_{p}^{g} \frac{M_{p}^{g-1}(t)}{T_{p}^{g-1}}-\frac{m_{p}^{g+1} M_{p}^{g}(t)}{T_{p}^{g}}+M_{p}^{g}(t)\right]\left[\frac{\mu_{p} M_{b}(t)}{K_{b}+M_{b}(t)}-\mu_{c p}\right] } \\
& -\left[r_{d_{p}}^{g}+\theta_{p}^{g}\right] M_{p}^{g}(\mathrm{t})+2 \sum_{\mathrm{i}=1}^{g} r_{d p}^{i} M_{p}^{i}(t) g_{2 p}^{i}\left(i_{1} g\right), \mathrm{g}=1,2,3,4,5 \\
\frac{d M_{b}(t)}{d t}= & \frac{\mu_{b} S_{u}(t)}{K_{u}+S_{u}(t)} M_{b}(t)-\theta_{b} M_{b}(t)-\frac{\mu_{b} M_{b}(t) M_{p}(t)}{W\left[K_{b}+M_{b}(t)\right]} \\
\frac{d S_{u}(t)}{d t}= & {\left[\theta_{b} \sigma_{1 b}+\mu_{c b} \alpha_{1 b}\right] M_{b}(t) } \\
& +\sum_{i=1}^{5}\left[\theta_{p}^{i} \sigma_{1 p}^{i}+\mu_{c p} \alpha_{1 p}^{i}\right] M_{p}^{i}(t)-\frac{\mu_{b} S_{u}(t)}{Y\left[K_{u}+S_{u}(t)\right]} M_{b}(t) \\
\frac{d S_{r}(t)}{d t}= & {\left[\theta_{b} \sigma_{2 b}+\mu_{c b} \alpha_{2 b}\right] M_{b}(t) } \\
& \quad 1 / 4 \sum_{i=1}^{5}\left(\theta_{p}^{i} \sigma_{2 p}^{i}+\mu_{c p} \alpha_{2 p}^{i}\right) M_{p}^{i}(t)
\end{aligned}
$$

In these equations, $\mathbf{M}_{\mathrm{p}}^{\mathrm{g}}(\mathrm{t})$ is the protozoan mass in the gth mass group; $M_{b}(t)$ is the total bacterial mass; $S_{u}(t)$ and $S_{r}(t)$ are the utilizable and refractory substrate concentrations, respectively; $\mathrm{M}_{\mathrm{p}} \mathrm{p}, \ldots, \mathrm{M}_{\mathrm{p}}^{\mathrm{b}}$ are the boundaries of each mass group which were chosen to coincide with those of the mass groups in the protozoan counter as shown in Fig. 8; and $T_{p}^{g}$ is defined as $T_{p}^{g}=M^{g+1}-M^{g}{ }_{p}$ which denontes the individual cell mass increment in group $\mathrm{g}$. The constants $\mu_{\mathrm{p}}$, $\mu_{\mathrm{cp}}$, and $\mathrm{K}_{\mathrm{b}}$ characterize the growth of a protozoan, and $\mu_{\mathrm{b}}, \mu_{\mathrm{cb}}$ and $\mathrm{K}_{\mathrm{u}}$ characterize the growth of a bacterium. In Eqs. (1) and (2) we have used a model for the growth of a single cell that takes into account saturation for substrate intake ( $\mathrm{K}_{\mathrm{b}}$ and $K_{u}$ are being the saturation constants) and endogenous metabolism, represented by $\mu_{\mathrm{cp}}$ and $\mu_{\mathrm{cb}}$. The constants $\mathrm{r}_{\mathrm{dp}}^{\mathrm{g}}$ and $\theta_{\mathrm{p}}^{\mathrm{g}}$ in (1) are the division and 


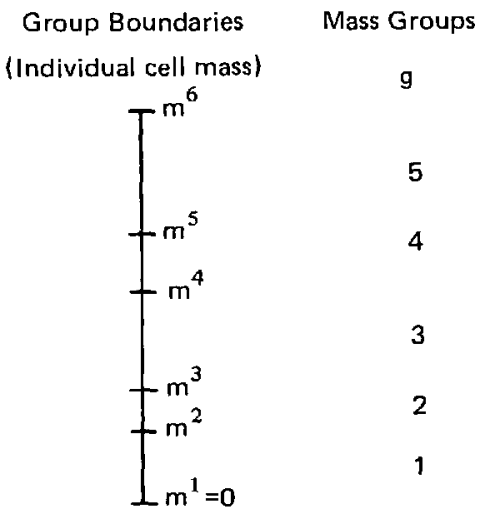

Fig. 8. Protozoan mass groups.

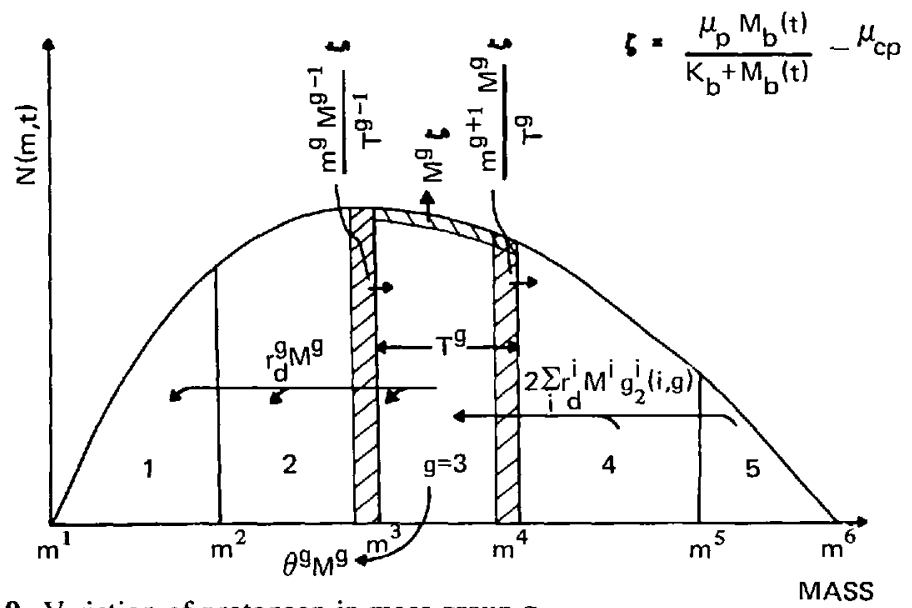

Fig. 9. Variation of protozoan in mass group $\mathrm{g}$.

death probabilities, respectively, per unit protozoan mass in group $g$ per second. The symbol $g_{z p}^{i}(i, g)$ is the probability that a unit of protozoan mass dividing in group $i$ produces a unit of protozoan in group $g$. It is zero for $i<g$ because a cell cannot produce a larger one on division. $W$ in (2) is the protozoan efficiency during consumption of bacteria. We assume here that the death and the endogeneous metabolism processes of the bacteria and protozoa produce fractions of utilizable as well as refractory substrate. The $\theta_{b}$ is the death probability per second of a bacterium. 
Figure 9 shows graphically the contribution of the individual terms in Eq. (1). When $g=1$ (the smallest cell group) the first term on the right, which gives the mass growth rate into this group from lower ones, vanishes because there are no smaller cells. When $g=5$ (the largest cell group) the second term on the right-hand side on Eq. (1), which gives the growth rate from this group to higher ones, vanishes because there are no larger cells.

In Eqs. (3) and (4) $\alpha_{1 \mathrm{~b}}$ and $\alpha_{\mathrm{lp}}^{\mathrm{i}}$ are the utilizable substrate fractions produced by the endogeneous metabolism of a bacterium and protozoan in the $i$ th group, respectively. Similarly $\alpha_{2 \mathrm{~b}}$ and $\alpha_{2 \mathrm{p}}^{i}$ are the refractory substrate fractions. The constants $\sigma_{1}$ and $\sigma_{2}$ are the utilizable and refractory substrate fractions, respectively, per unit mass of a dead cell. In Eq. (3) $Y$ is the bacterial yield efficiency during substrate consumption.

The derivation of the above equations and a more precise definition of various parameters in them have been presented in Villarreal et al [3]. However, they can also be obtained directly from the mass balance of protozoan in each group, bacterium, and utilizable and refractory substrate concentrations, with an appropriate model for the growth of an individual cell.

The numerical integration of these equations in a computer is fairly standard. We solved them using a modified predictor-corrector method [5], with initial conditions that approximated the experimental conditions at the initial time.

The values of the parameters used in the numerical calculations were obtained using the experimental results from batch and continuous flow reactors reported by Lustig [6] and the results of batch runs presented herein. In all cases the reactors contained Tetrahymena pyriformis grown monoxenically on cultures of Aerobacter aerogenes. The parameter values given in Tables 1 and 2 have been used for the numerical calculations in the present paper and constitute a slightly improved version of the ones given to reproduce earlier experiments [3]. The procedure to obtain the numbers in Table 1 was essentially one of trial and error, although $r, \theta, \alpha$, and $\sigma$ were obtained from the decay curves of bacteria. The group constants in Table 2 were obtained using decay curves in the case of $\alpha, \sigma$, $r$, and $\theta$, but the last two are variable according to their definition. The values of $m$ and $\mathrm{m}$ were obtained from the particle counter, and the values of $g_{2 \mathrm{p}}$ assume a trianglar distribution around half the parent mass cell for the probability of a newborn cell.

\section{Discussion}

The results of the numerical integrations are presented in Fig. 1 through 7 for batch runs II and III. In the first of these two runs the inoculum contained mostly large cells, in the last run it contained a majority of small cells. We find that the experimental curves are reproduced fairly well by the numerical integra- 
Table 1

Parameter Values

$$
\begin{array}{rlrl}
\mu_{b} & =0.515 \mathrm{hr}^{-1} & \mu_{p} & =0.134 \mathrm{hr}^{-1} \\
\mu_{c b} & =0.0036 \mathrm{hr}^{-1} & \mu_{c p} & =0.586 \mathrm{hr}^{-1} \\
K_{u} & =1.55 \mathrm{mg} / \mathrm{liter} & K_{b} & =0.25 \mathrm{mg} / \mathrm{liter} \\
Y & =0.428 \mathrm{gm} \text { of } b / \mathrm{gm} \text { of } S_{u} & W & =0.75 \mathrm{gm} \text { of } b / \mathrm{g} \text { of } p \\
\theta_{b} & =0.0036 \mathrm{hr}^{-1} & \theta_{p} & =0.007 \mathrm{hr} \\
\sigma_{1 b} & =0.556 \mathrm{gm} \text { of } S_{u} / \mathrm{gm} \text { of } b & r_{p} & =0.022 \mathrm{hr} \\
\sigma_{2 b} & =0.1665 \mathrm{gm} \text { of } S_{r} / \mathrm{gm} \text { of } b & \sigma_{1 p} & =0.2 \mathrm{gm} \mathrm{of} S_{u} / \mathrm{gm} \text { of } p \\
a_{1 b} & =0.6 \mathrm{gm} \text { of } S_{u} / \mathrm{gm} \text { of } b & a_{2 p} & =0.43 \mathrm{gm} \text { of } S_{r} / \mathrm{gm} \text { of } p \\
a_{2 b} & =0.1 \mathrm{gm} \text { of } S_{r} / \mathrm{gm} \text { of } b \quad a_{2 p}=0.25 \mathrm{gm} \text { of } S_{r} / \mathrm{gm} \text { of } p
\end{array}
$$

Table 2

Five Group Constants for Protozoa

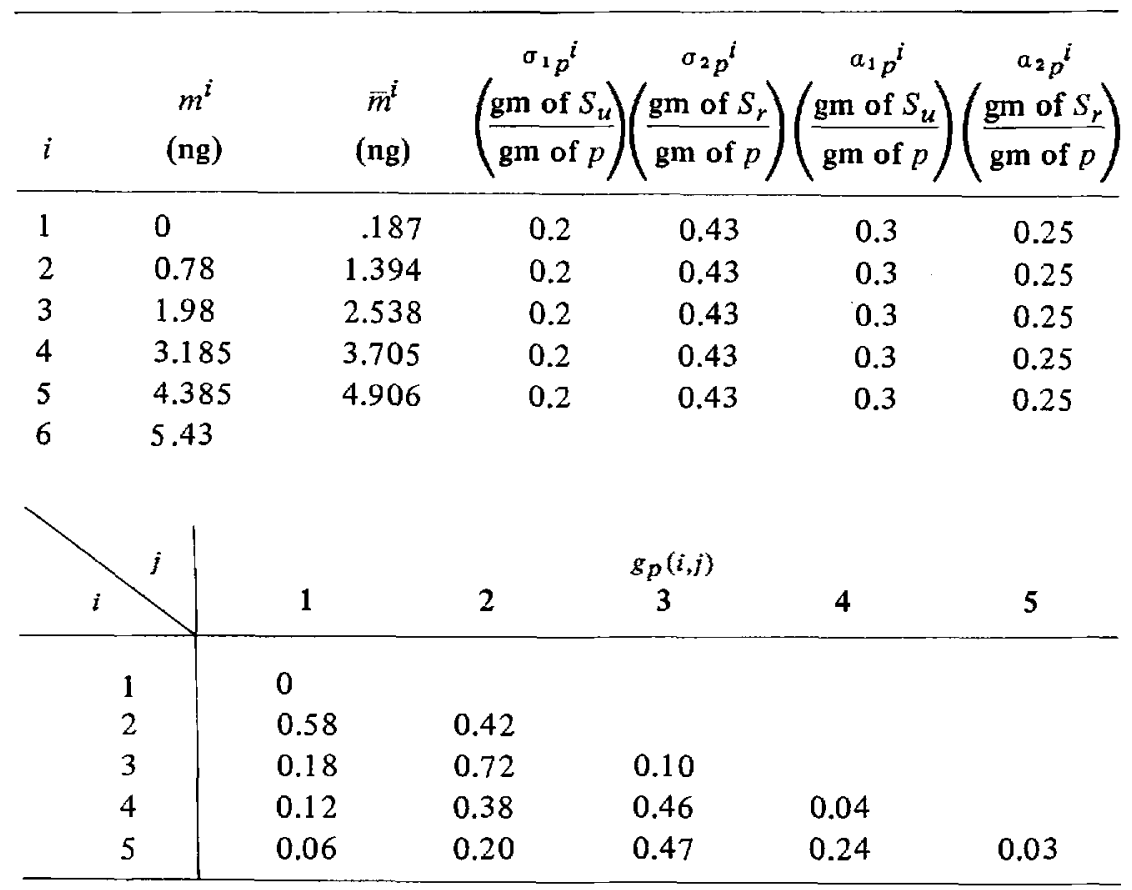


tions. For most groups the number of predators in batch reactor II is larger during the first half of the experiment than for batch reactor III. This is true experimentally as well as numerically. We note that the predicted curves for group one (small protozoan) are inverted; that is, the calculated curve for batch run II should be above the one for batch run III. It is also noted that the growth of the ciliates is faster in batch run II than in batch run III, apparently due to the difference in the inoculum. Large predators are more apt to initiate growth.

Although the match between the experiments and the model is encouraging, the assumptions of the present theory certainly need further testing. In this respect one should try to answer experimentally several questions. How reproducible are the experiments? Are individual cell mass data sufficient to characterize the growth of these microbial species? And if so, is the Monod type model with an endogenous metabloism term acceptable for the description of the growth of a cell? Even if all the assumptions we have made are valid, we need detailed information about the mass dependence of division probabilities, death probabilities, distribution of daughter cells, perhaps growth coefficients, etc., although the values we use do not seem to be very unrealistic.

We have shown in this paper that by using multigroup theory one can solve numerically the equations describing the transients in mass distributions of species in a changing environment and predict the results of batch experiments under various initial conditions of variable starting size for the protozoan predator. The biological parameters appearing in the theory can be obtained from a comparison of the theoretical and experimental results by using a set of welldesigned experiments and some mathematical analysis, as suggested by Von Foerster [7]. Knowledge of these parameters shed light on the behavior of the individual cells in changing environments. The extension of this theoretical approach to the investigation of other variables besides mass, such as the cell volume and space dependence, is only a matter of mathematical exercise in the formulation [4]. For example, Von Foerster [7] has analyzed extensively equations for the particular case of age dependence. The difficulty, however, lies in the evaluation of the parameters appearing in such a detailed description. In this respect, one perhaps needs more accurate experiments on the transients of interacting populations under a wider range of experimental conditions, as well as experiments involving the growth of single cells in a changing environment.

\section{Acknowledgments}

This work is part of a Ph.D. thesis by Ernesto Villarreal. The work was supported by the Nuclear Engineering Department of the University of Michigan. The experiments were supported by the Sea Grant Program at the same University. The authors express their appreciation for the help with the experimental phases of this paper given to them by Mr. James Graham of the Zoology Department. 


\section{References}

1. Canale, R.P., Lustig, T.D., Kehrberger, P.M., and Salo, J.E. 1973. Experimental and mathematical modeling studies of protozoan predation on bacteria. Biotech. and Bioeng., 15: 107.

2. Curds, C.R. 1971. Computer simulations of microbial population dynamics in the activated sludge process. Water Res. 5: 1049.

3. Villarreal, E., Akcasu, A., and Canale, R. 1976. A theory of interacting microbial populations: Multigroup approach. J. of Theor. Biol. 58: 285-317.

4. Villarreal, E., Canale, R., and Akcasu, A. 1975. A multigroup model for predator-prey interactions. Biotech. and Bioeng. 17, 1269.

5. Villarreal, E. 1974. A theory on interacting species: A multigroup approach to microbial growth. Ph.D. Thesis. The University of Michigan.

6. Lustig, T.D. 1971. Predator-prey interactions in an experimental protozoan community (Tetrahymena pyriformis-Aerobacter aerogenes). Technical Report of The University of Michigan Sea Grant Program. Ann Arbor, Michigan.

7. Von Foerster, H. 1959. Some remarks on changing populations. In: Kinetics of Cellular Proliferation. R. Stohlman, Jr., editor. Grune and Stratton. N.Y., N.Y. 